\title{
Roteamento Adaptativo e Agregação de Tráfego em Redes Ópticas WDM Dinâmicas
}

\author{
Paulo Ribeiro Lins Júnior, Marcelo Sampaio de Alencar e Edmar Candeia Gurjão
}

\begin{abstract}
Resumo-Este trabalho trata do estabelecimento dinâmico de caminhos ópticos em redes WDM, considerando duas técnicas de engenharia de tráfego: o balanceamento de carga utilizando roteamento adaptativo, na qual são estudadas cinco funções custo, e a agregação de tráfego. As técnicas têm seus desempenhos comparados com relação à probabilidade de bloqueio e à utilização dos enlaces, para duas topologias. Os resultados mostram que a integração do roteamento adaptativo com a agregação de tráfego para o roteamento e alocação de comprimento de onda melhora o desempenho com relação à probabilidade de bloqueio e à distribuição de carga entre os enlaces da rede.
\end{abstract}

Palavras-Chave-Redes Ópticas WDM, Engenharia de Tráfego, Roteamento Adaptativo, Agregação de Tráfego

Abstract-This work discusses the dynamic routing and wavelength assignment in WDM networks. Two traffic engineering strategies are considered: load balancing, using adaptive routing, in this five weigh functions are studied, and traffic grooming. The strategies are compared in terms of blocking probability link average utilization, to two topologies. The results show that integration of adaptive routing algorithm with traffic grooming for routing and wavelength assignment improves better performance with respect to blocking probability and load distribution between the links of the network.

Keywords-WDM Optical Networks, Traffic Engineering, Adaptive Routing, Traffic Grooming

\section{INTRODUÇÃO}

As redes ópticas multiplexadas por divisão em comprimento de onda (WDM - Wavelength Division Multiplexing), devido, principalmente, às suas características físicas, tem ganhado cada vez mais aceitação como meio de transporte promissor tanto para o tráfego da Internet quanto para outras fontes de tráfego que requerem qualidade de serviço.

Os usuários dessas redes se comunicam por conexões ópticas estabelecidas fim-a-fim, denominadas caminhos ópticos e que utilizam, na ausência de conversão de comprimento de onda, o mesmo comprimento de onda disponível em todos os enlaces que compõem o caminho. Tal exigência é conhecida como restrição de comprimento de onda [1].

Nessas redes utiliza-se um plano de controle que tem conhecimento atualizado do estado da rede e coordena a tarefa de selecionar rotas e comprimentos de onda disponíveis para o estabelecimento de um caminho óptico. Também é do plano de controle a responsabilidade de garantir que as conexões estabelecidas nesses caminhos ópticos tenham a qualidade

Paulo Ribeiro Lins Júnior, Marcelo Sampaio de Alencar e Edmar Candeia Gurjão, Instituto de Estudos Avançados em Comunicações - IECOM, Departamento de Engenharia Elétrica, Universidade Federal de Campina Grande, Campina Grande - PB, Brasil, E-mails: paulo.ribeiro.lins.jr@gmail.com, malencar@iecom.org.br, ecandeia@dee.ufcg.edu.br. Este trabalho foi fomentado pela CAPES. de serviço solicitada pelos usuários da rede. Nesse contexto, a engenharia de tráfego surge como a principal ferramenta capaz de proporcionar a garantia de qualidade solicitada pelos usuários [2] [3].

Dentre as possíveis ferramentas da engenharia de tráfego para redes WDM, duas são abordadas neste trabalho: o roteamento adaptativo e a agregação de tráfego. Para o roteamento adaptativo, são comparados os desempenhos de cinco propostas de funções custo. O desempenho dessas funções é avaliado com relação ao número de bloqueios ocorridos na rede durante um determinado período de tempo e com relação à ocupação de cada enlace pelas conexões estabelecidas. A proposta apresentada sugere a integração de um algoritmo de agregação de tráfego em redes ópticas a ser usado em conjunto com o algoritmo adaptativo proposto, na tentativa de obter um melhor balanceamento da carga na rede, diminuindo, assim, a sobrecarga em determinados enlaces e proporcionando o uso mais uniforme dos recursos disponíveis.

O artigo é apresentado da seguinte forma: na seção II são apresentados o problema do roteamento adaptativo e as funções custo avaliadas neste trabalho; na seção III, é analisada a capacidade de agregação de tráfego em nós ópticos e é apresentado o algoritmo de agregação de tráfego implementado; na seção IV é descrito o ambiente de simulação e são apresentados e discutidos os resultados obtidos nos experimentos realizados; na seção V são feitas as conclusões obtidas com base nos resultados conseguidos.

\section{RWA ADAPTATIVO}

O problema do roteamento e alocação de comprimento de onda pode ser apresentado da seguinte forma. Considere uma rede com $M$ nós, $K$ enlaces e $W$ comprimentos de onda. $\mathrm{O}$ estado do $i$-ésimo enlace, com $1 \leqslant i \leqslant K$, no instante de tempo $t$ pode ser especificado pelo vetor coluna

$$
\sigma_{t}^{(i)}=\left[\begin{array}{c}
\sigma_{t}^{(i)}(1) \\
\sigma_{t}^{(i)}(2) \\
\vdots \\
\sigma_{t}^{(i)}(W)
\end{array}\right],
$$

em que, $\forall j$ tal que $1 \leqslant j \leqslant W, \sigma_{t}^{(i)}(j)=1$ se o comprimento de onda $j$ é usado por um caminho óptico no instante de tempo $t$, no enlace $i$ e $\sigma_{t}^{(i)}(j)=0$ se estiver disponível. Assim sendo, o estado da rede é descrito pela matriz 


$$
\sigma_{t}=\left[\begin{array}{cccc}
\sigma_{t}^{(1)}(1) & \sigma_{t}^{(2)}(1) & \cdots & \sigma_{t}^{(K)}(1) \\
\sigma_{t}^{(1)}(2) & \sigma_{t}^{(2)}(2) & \cdots & \sigma_{t}^{(K)}(2) \\
\vdots & \vdots & \ddots & \vdots \\
\sigma_{t}^{(1)}(W) & \sigma_{t}^{(2)}(W) & \cdots & \sigma_{t}^{(K)}(W)
\end{array}\right] .
$$

Os enlaces são indexados por um número $\mathrm{i}, i \in\{1, \ldots, K\}$, e o enlace $e_{j}$ representa a ligação entre dois nós da rede, sem que necessariamente um deles seja o nó $j$. Dada uma requisição para o estabelecimento de conexão no instante de tempo $t$, a função do algoritmo de RWA é selecionar um caminho $E$, composto pelos enlaces $\left(e_{1}, e_{2}, \ldots, e_{n}\right)$ que interligam os nós origem e destino, tal que $\sigma_{t}^{\left(e_{l}\right)}(j)=0$ para todo $l=1,2, \ldots, n$. Tal consideração satisfaz a restrição de continuidade de comprimento de onda.

O algoritmo proposto nesse trabalho considera todos as possíveis rotas entre um par de nós e utiliza a informação do estado atual da rede para ponderar as rotas, de forma que a rota em melhores condições esteja no topo da lista das possíveis rotas. Uma abordagem similar é usada por [4] com uma comparação entre três algoritmos clássicos de roteamento - o RIP (Routing Information Protocol), o OSPF (Open Shortest Path Function) e o IGRP (Interior Gateway Routing Protocol) - e o algoritmo WLC (Weighted Link Capacity) proposto no trabalho. Em [5], os autores abordam o balanceamento de carga sendo executado a partir de modificações na tabela de roteamento, na tentativa de se obter a melhor rota. Essa rota é analisada e buscada em um conjunto das rotas possíveis e seu uso configura, segundo o trabalho, a necessidade de modificação na tabela. Em [6], os autores realizam um estudo comparativo entre três métricas de roteamento adaptativo, duas das quais serão consideradas neste trabalho.

Para o roteamento foi utilizada uma adaptação do algoritmo de Dijkstra, como descrito em [6]. Esse algoritmo possui uma natureza aditiva, pois para um caminho com $L$ enlaces $P=$ $\left\{e_{1}, e_{2}, \ldots, e_{L}\right\}, \forall e_{i} \in E$, sendo $i \in\{1,2,3, \ldots, K\}, \mathrm{o}$ custo total do caminho $P$ é calculado como o somatório dos custos individuais de cada enlace que o compõem, ou seja,

$$
C_{T, P}=\sum_{i=1}^{L} C_{e_{j}, P}
$$

em que $C_{T, P}$ representa o custo total da rota $P$ e $C_{e_{i}, P}$ indica o custo do enlace individual $e_{i}$, que compõe a rota $P$.

Neste trabalho, convencionou-se que, para um enlace entre os nós $i$ e $j$, ao qual está associado um índice $e_{l}, C_{i j}$ denota o custo do enlace entre os nós $i$ e $j$ na $k$-ésima iteração; $C_{i j}^{-1}$ denota o custo do enlace entre os nós $i$ e $j$ na iteração anterior à $k$-ésima iteração; $\lambda_{i j}^{U}$ refere-se à quantidade de comprimentos de onda utilizados no enlace e $\lambda_{i j}^{T}$ representa o número total de comprimentos de onda no enlace.

\section{A. Funções Custo Avaliadas}

As funções custo $C_{i j}$ consideradas nesse estudo são:

Baseada no número de enlaces (NE) - Nesse caso o custo é dado por

$$
C_{i j}=1, \forall(i, j) \in E \text {. }
$$

Esse representa o caso mais básico, em que o roteamento não é adaptativo. Essa função é adotada para fins de comparação de desempenho entre as demais relações propostas.

Baseada na capacidade do enlace 1 (CE1) - A função custo, $\forall(i, j) \in E$, é dada por

$C_{i j}= \begin{cases}C_{i j}^{-1}+1, & \text { se uma nova conexão foi estabelecida } \\ C_{i j}^{-1}-1, & \text { se uma conexão ativa foi finalizada. }\end{cases}$

Baseada na capacidade do enlace 2 (CE2) - Essa função custo, apresentada em [4], é especificada, $\forall(i, j) \in E$, da seguinte forma

$$
C_{i j}=\left\{\begin{array}{cc}
\frac{\lambda_{i j}^{T}}{\lambda_{i j}^{T}-\lambda_{i j}^{U}} & \text { se } \lambda_{i j}^{U}<\lambda_{i j}^{T}, \\
\infty & \text { se } \lambda_{i j}^{U}=\lambda_{i j}^{T} .
\end{array}\right.
$$

Nessa função, o valor do peso aumenta com características exponenciais à medida que $\lambda_{i j}^{U}$ aumenta. Isto indica que a estratégia de roteamento deve evitar a seleção de um enlace cuja variável $\lambda_{i j}^{U}$ tenha aumentado, o que pode levar à esperada distribuição do tráfego entre todos os enlaces da rede.

Baseada na capacidade do enlace 3 (CE3) - Essa função custo é baseada na função apresentada por [6] e é especificada como

$$
C_{i j}=\left\{\begin{array}{cc}
1-\log \left[\left(1-\frac{\lambda_{i j}^{U}}{\lambda_{i j}^{T}}\right)^{\lambda_{i j}^{U}}\right] & \text { se } \lambda_{i j}^{U}<\lambda_{i j}^{T}, \\
\infty & \text { se } \lambda_{i j}^{U}=\lambda_{i j}^{T} .
\end{array}\right.
$$

Para evitar a influência dessa natureza aditiva, é utilizada a abordagem baseada no logarítmo como função custo.

Baseada na capacidade do enlace 4 (CE4) - Essa função é proposta neste trabalho como alternativa às funções acima descritas. O custo é alterado da seguinte maneira

$$
C_{i j}=1+\lambda_{i j}^{U} \cdot \exp \left(\frac{\lambda_{i j}^{U}}{\lambda_{i j}^{T}}\right) .
$$

A motivação para a escolha de uma função exponencial se deve ao fato de que além de evitar uma alternativa à natureza aditiva, apresentar uma característica de convergência distinta da função logarítma.

\section{Agregação DE TrÁfego}

O estabelecimento eficiente das conexões com taxas de transmissão diversificadas e inferiores à capacidade do canal é um importante problema da Engenharia de Tráfego, conhecido como problema de agregação de tráfego [7].

Alguns trabalhos propõem também o estudo de agregação considerando um padrão de tráfego dinâmico em redes ópticas WDM. Em [8] são comparados dois algoritmos para seleção de rotas e seus resultados indicam que, para se obter um desempenho aceitável em um ambiente dinâmico, deve-se usar diferentes abordagens para a agregação de tráfego e para a seleção de rotas em diferentes estados da rede. Em [9], os autores propõem um esquema para controle de admissão de conexão 
para assegurar uma probabilidade de bloqueio menor e mais distribuída na rede. Um modelo de correlação entre capacidade do enlace e capacidade de agregação dos nós é apresentado por [10] para calcular a probabilidade de bloqueio em uma rede WDM com restrições na capacidade de agregação. O trabalho de [11] apresenta esquemas de estabelecimento de caminhos ópticos em redes ópticas em malha considerando a capacidade de agregação de tráfego na rede. Em [12] são estudados o planejamento e o projeto de redes WDM em malha com previsão da demanda de tráfego, com o intuito de minimizar os custos associados à distribuição e utilização dos recursos disponíveis e satisfazer todas as conexões. Em [13] desenvolveu-se uma heurística para agregação de tráfego baseada no conceito de colaboração de grupo.

\section{A. Alocação de Comprimento de Onda com Agregação de Tráfego}

O algoritmo de agregação de tráfego proposto neste trabalho baseia-se no algoritmo conhecido como Direct-link [14], que tem como diretriz primária maximizar a utilização dos comprimentos de onda. $\mathrm{O}$ algoritmo aqui proposto procura, para toda requisição de estabelecimento de um caminho óptico, um canal óptico estabelecido que satisfaça a demanda por banda da requisição. Se não existir caminho óptico estabelecido com banda disponível suficiente para tal, então tenta-se o estabelecimento de um novo canal. No caso da capacidade de transmissão do comprimento de onda testado ser inferior à requerida pela conexão, os comprimentos de onda seguintes são testados até que se encontre um com capacidade suficiente ou não se encontre nenhum, caracterizando situação de bloqueio na rota. Esse algoritmo se apresenta como uma modificação do First-Fit convencional, descrito em [1] e é apresentado no algoritmo 1 .

Para a descrição do algoritmo, são utilizadas as seguintes definições: $P_{l}$ : vetor com os enlaces que compõe o caminho óptico entre $i$ e $j ; L$ : comprimento do caminho óptico, ou seja, número de enlaces existentes na menor rota entre o nós origem e destino; $\lambda(i j)$ : elemento da matriz de ocupação, indexada pelo número do enlace $i$ e pelo comprimento de onda $j$. $\mathrm{O}$ valor $\lambda(i j)=1$ indica que o comprimento de onda $j$ do enlace $i$ está ocupado, enquanto que o valor $\lambda(i j)=0$, qualquer que seja o enlace $i$, indica que o comprimento de onda $j$ está livre; $\lambda_{M A X}$ : número máximo de comprimentos de onda por enlace; $B_{i j}^{T}$ : largura de banda disponível no comprimento de onda $j$ do enlace $i ; B_{i}^{k}$ : taxa de transmissão ou largura de banda que deverá ser ocupada pela $k$-ésima conexão sobre o enlace $i$. É fácil observar que $B_{i}^{k} \leqslant B_{i}^{T} ; w$ : índice do comprimento de onda; LambdaAlocado: variável utilizada para controle do algoritmo; temp: variável temporária.

\section{Simulação E Resultados}

\section{A. Ambiente de simulação}

Para a avaliação dos algoritmos, foram realizadas simulações. São consideradas nos experimentos duas topologias de rede em malha, uma malha de seis nós, mostrada na Fig. 1 e uma segunda, baseada na rede da National Science Foundation, conhecida como NSF, apresentada na Fig. 2 em dois

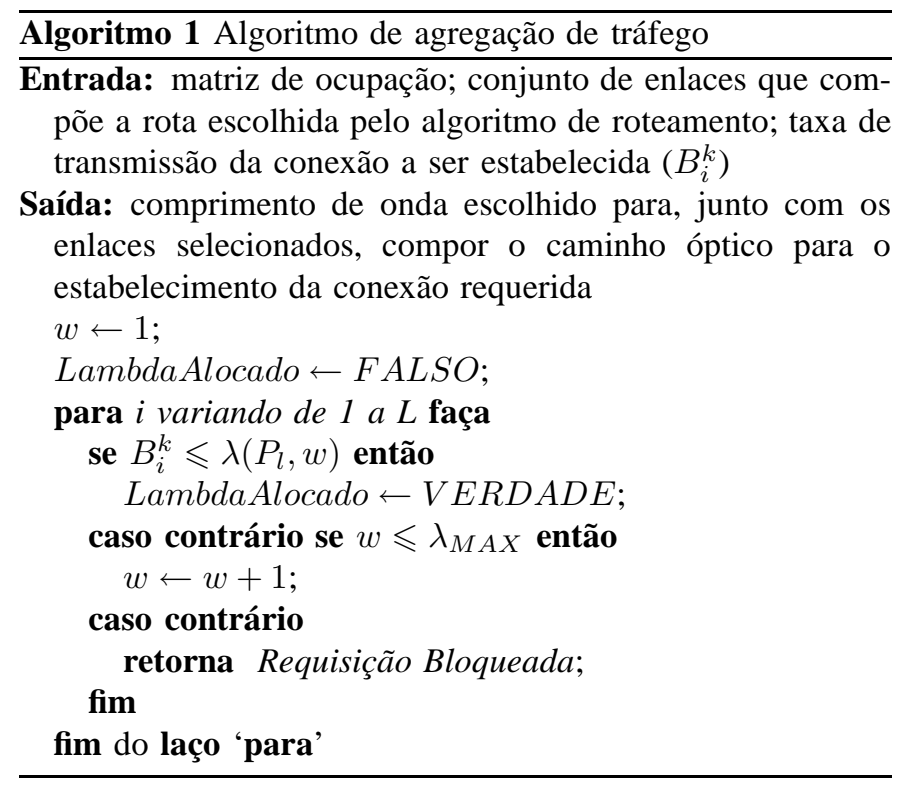

cenários de simulação: um considerando o uso de roteamento adaptativo sem agregação de tráfego e outro considerando o uso das duas técnicas conjuntamente.

A carga na rede varia de 200 a 800 erlangs. O tempo médio entre a requisição de conexões segue a distribuição de Poisson com média fixa de uma unidade de tempo e o tempo médio de duração das conexões estabelecidas segue uma distribuição exponencial negativa, com média variando de 200 a 800 unidades de tempo, com incremento de 30 unidades, o que perfaz um montante de 21 valores diferentes de carga considerados.

Cada enlace da rede contém duas fibras unidirecionais, com direções opostas, cada uma contendo 24 comprimentos de onda. Cada comprimento de onda tem uma capacidade de $10 \mathrm{Gbits} / \mathrm{s}$ e cada conexão pode ter uma taxa de transmissão variando de $1 \mathrm{G} b i t s / \mathrm{s}$ até $10 \mathrm{G} b i t s / \mathrm{s}$.

São adotadas duas métricas de desempenho: a probabilidade de bloqueio, definida como a razão entre o número de bloqueios ocorridos sobre o número de requisições efetuadas em toda a rede e a utilização média dos enlaces, definida como a razão entre o número de requisições estabelecidas em um dado enlace, e o número de requisições estabelecidas em toda a rede. Para a realização do estudo, a utilização do enlace é calculada para um valor baixo de carga, visando uma melhor análise da distribuição dos caminhos ópticos pela rede.

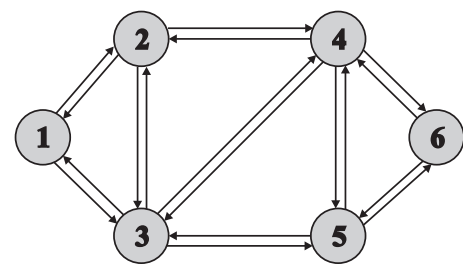

Fig. 1. Topologia de seis nós.

Os resultados obtidos para a utilização média dos enlaces são mostrados em um gráfico da porcentagem de conexões 


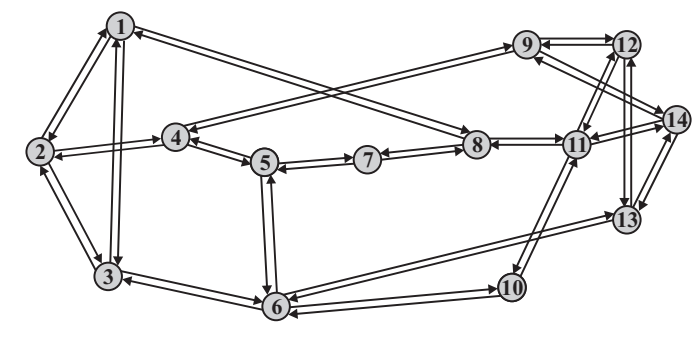

Fig. 2. Topologia da rede NSF.

estabelecidas para cada enlace da rede. Esses enlaces são indexados de acordo com as Tabelas I e II, para as redes com topologia de seis nós e NSF, respectivamente.

TABELA I

ÍNDICES DOS ENLACES DA TOPOLOGIA DE SEIS NÓS.

\begin{tabular}{|c|c||c|c||c|c|}
\hline Índice & Enlace & Índice & Enlace & Índice & Enlace \\
\hline \hline 1 & $1-2$ & 7 & $3-2$ & 13 & $4-6$ \\
\hline 2 & $1-3$ & 8 & $3-4$ & 14 & $5-3$ \\
\hline 3 & $2-1$ & 9 & $3-5$ & 15 & $5-4$ \\
\hline 4 & $2-3$ & 10 & $4-2$ & 16 & $5-6$ \\
\hline 5 & $2-4$ & 11 & $4-3$ & 17 & $6-4$ \\
\hline 6 & $3-1$ & 12 & $4-5$ & 18 & $6-5$ \\
\hline
\end{tabular}

TABELA II

ÍNDICES DOS ENLACES DA TOPOLOGIA DA REDE NSF.

\begin{tabular}{|c|c||c|c||c|c|}
\hline Índice & Enlace & Índice & Enlace & Índice & Enlace \\
\hline \hline 1 & $1-2$ & 15 & $5-7$ & 29 & $10-11$ \\
\hline 2 & $1-3$ & 16 & $6-3$ & 30 & $11-8$ \\
\hline 3 & $1-8$ & 17 & $6-5$ & 31 & $11-10$ \\
\hline 4 & $2-1$ & 18 & $6-10$ & 32 & $11-12$ \\
\hline 5 & $2-3$ & 19 & $6-13$ & 33 & $11-14$ \\
\hline 6 & $2-4$ & 20 & $7-5$ & 34 & $12-9$ \\
\hline 7 & $3-1$ & 21 & $7-8$ & 35 & $12-11$ \\
\hline 8 & $3-2$ & 22 & $8-1$ & 36 & $12-13$ \\
\hline 9 & $3-6$ & 23 & $8-7$ & 37 & $13-6$ \\
\hline 10 & $4-2$ & 24 & $8-11$ & 38 & $13-12$ \\
\hline 11 & $4-5$ & 25 & $9-4$ & 39 & $13-14$ \\
\hline 12 & $4-9$ & 26 & $9-12$ & 40 & $14-9$ \\
\hline 13 & $5-4$ & 27 & $9-14$ & 41 & $14-11$ \\
\hline 14 & $5-6$ & 28 & $10-6$ & 42 & $14-13$ \\
\hline
\end{tabular}

Para cada valor de carga, são feitas 20.000 conexões, em um total de cinco execuções. Devido à natureza dinâmica do tráfego, para os cálculos da probabilidade de bloqueio e da ocupação dos enlaces, considera-se a média dos resultados parciais de cada uma das execuções.

\section{B. Resultados obtidos}

Para o primeiro cenário, considera-se o uso de roteamento adaptativo, utilizando as funções custo descritas, sem a capacidade de agregação de tráfego nos nós da rede.

O gráfico da probabilidade de bloqueio em função da carga oferecida na rede é apresentado na Fig. 3, para a topologia de seis nós e na Fig. 4, para a topologia da rede NSF.

Para a rede de seis nós (Fig. 3) os bloqueios começam a acontecer, considerando a estratégia adaptativa para o roteamento, com uma carga de 330 erlangs. Sem roteamento

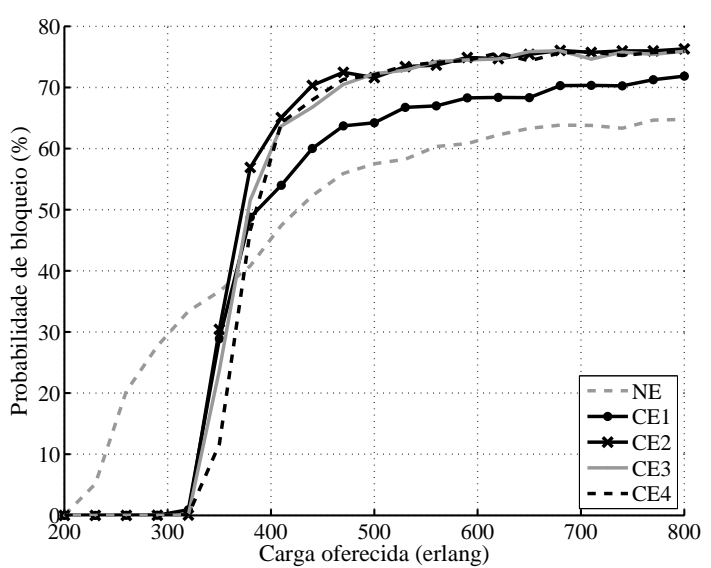

Fig. 3. Probabilidade de bloqueio em função da carga oferecida para a topologia de seis nós no $1^{\mathrm{o}}$ cenário.

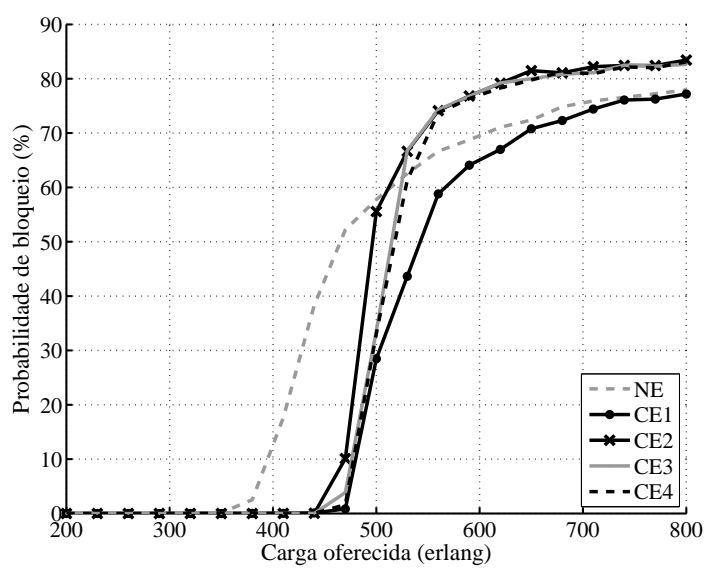

Fig. 4. Probabilidade de bloqueio em função da carga oferecida para a topologia da rede NSF no $1^{\circ}$ cenário.

adaptativo, os bloqueios começam a acontecer com uma carga de 200 erlangs. Na faixa entre 200 erlangs e 350 erlangs, o roteamento adaptativo tem uma vantagem considerável sobre o não adaptativo. Acima de 350 erlangs, porém, a estratégia de roteamento adaptativa deixa de ser vantajosa, tendo em vista que a probabilidade de bloqueio obtida é aproximadamente $12 \%$ superior. Dentre as funções custo, duas se destacam. Até uma carga de 380 erlangs, a função CE4, proposta nesse trabalho, possui o melhor desempenho, ao passo que, após essa carga, a função CE1 se destaca com uma melhoria considerável na probabilidade de bloqueio com relação às outras funções.

Para a rede NSF (Fig. 4), os bloqueios começam a acontecer com uma carga de 350 erlangs para o roteamento não adaptativo e 470 erlangs, para o adaptativo. Devido à maior quantidade de nós e enlaces, os bloqueios nessa topologia acontecem com uma carga maior se comparados à topologia de seis nós.

O resultado da utilização dos enlaces é mostrado no gráfico do número de conexões estabelecidas para cada enlace da rede, de acordo com a indexação apresentada nas Tab. I e II, para a topologia de seis nós na Fig. 5 e para a topologia da rede 
NSF na Fig. 6.

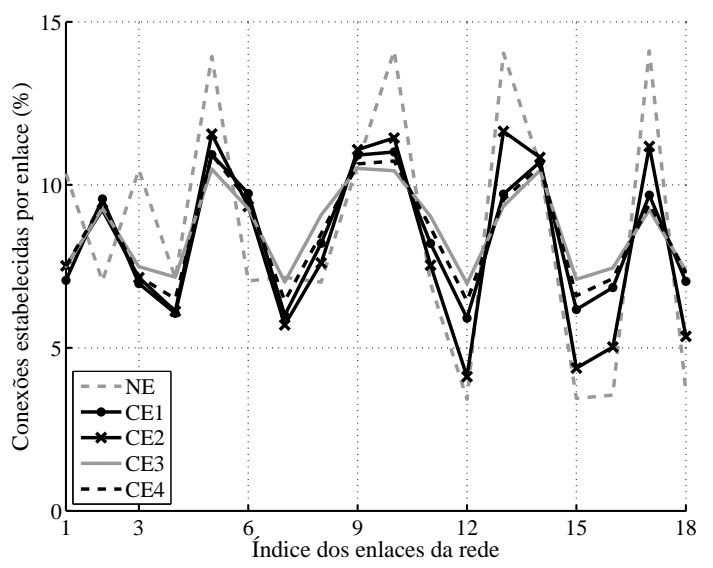

Fig. 5. Porcentagem de conexões estabelecidas para cada enlace para a topologia de 6 nós no $1^{\circ}$ cenário.

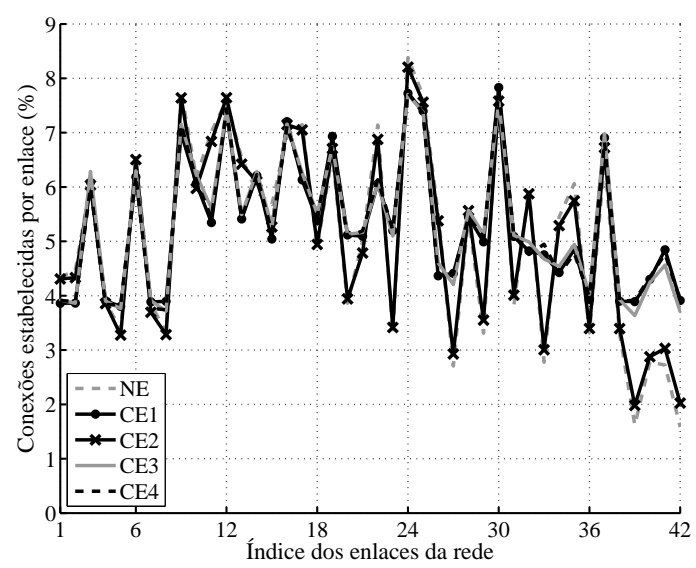

Fig. 6. Porcentagem de conexões estabelecidas para cada enlace para a topologia da rede NSF no $1^{\circ}$ cenário.

Com relação à porcentagem de conexões estabelecidas em cada enlace para a topologia de seis nós (Fig. 5), o uso de uma abordagem adaptativa para o roteamento melhorou a distribuição do tráfego para os enlaces, diminuindo os picos e vales observados pelo resultado da função NE. Esse resultado se deve ao fato de que o roteamento adaptativo altera o valor dos custos, desestimulando o uso dos enlaces que possuem mais conexões ativas e, por conseguinte, mais comprimentos de onda utilizados. Dentre as funções custo estudadas no roteamento adaptativo, as funções CE1, CE3 e CE4 possuem um desempenho praticamente similar, sendo superior ao apresentado pela função CE2.

Para a rede NSF, conforme mostrado na Fig. 6, a utilização dos enlaces não se altera com o uso de roteamento adaptativo comparativamente à abordagem sem atualização da tabela de roteamento. Esse resultado pode ser justificado com base na maior quantidade de nós e de enlaces dessa topologia. O maior número de enlaces leva à uma maior quantidade de opções de rotas a serem utilizadas entre qualquer par de nós origem e destino. Por conseguinte, as conexões, mesmo sem o uso de roteamento adaptativo, tendem a se distribuir pela rede de uma maneira mais uniforme para essa topologia do que para as outras estudadas.

O segundo cenário tem como objetivo comparar o desempenho do algoritmo de agregação de tráfego com a consideração das funções custo avaliadas.

O gráfico da probabilidade de bloqueio em função da carga oferecida na rede é apresentado na Fig. 7, para a topologia de seis nós e na Fig. 8, para a topologia da rede NSF.

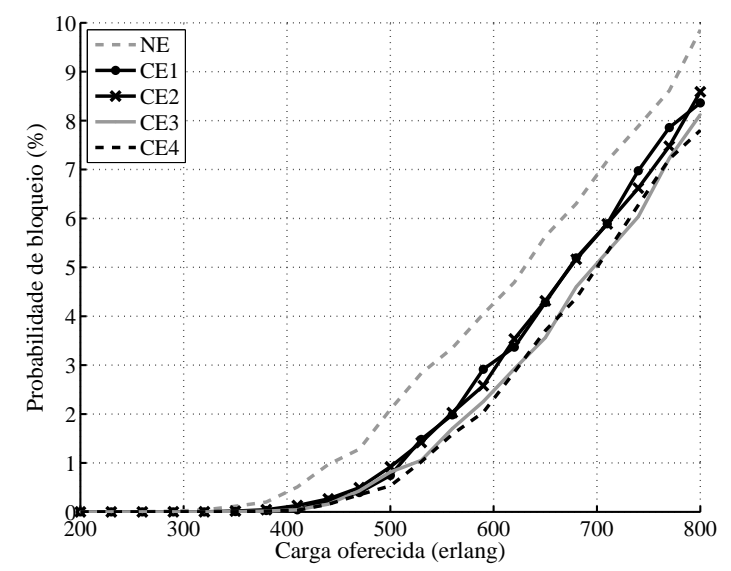

Fig. 7. Probabilidade de Bloqueio em função da carga oferecida para a topologia de seis nós no $2^{\circ}$ cenário.

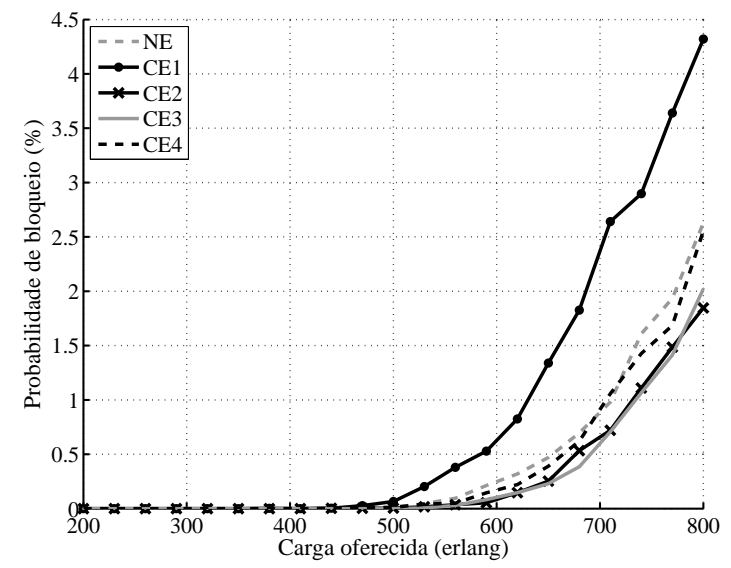

Fig. 8. Probabilidade de Bloqueio em função da carga oferecida para a topologia da rede NSF no $2^{\circ}$ cenário.

Os resultados apresentados mostram os bloqueios começam a ocorrer com cargas mais altas e, na faixa de carga analisada, o roteamento adaptativo acarreta uma probabilidade de bloqueios sempre inferior ao roteamento não adaptativo, mostrando melhor desempenho com relação ao primeiro cenário. Para a topologia de seis nós, as funções custo possuem um desempenho praticamente igual, tendo apenas, para a rede de seis nós, uma ligeira vantagem das funções custo CE3 e CE4. Para a topologia da rede NSF, observa-se, no entendo, que apenas as funções custo CE2 e CE3 ocasionaram uma pequena vantagem sobre o uso do roteamento não adaptativo.

A porcentagem de utilização dos enlaces para o segundo 
cenário é mostrada, para a topologia de seis nós, na Fig. 9 e para a topologia da rede NSF, na Fig. 10.

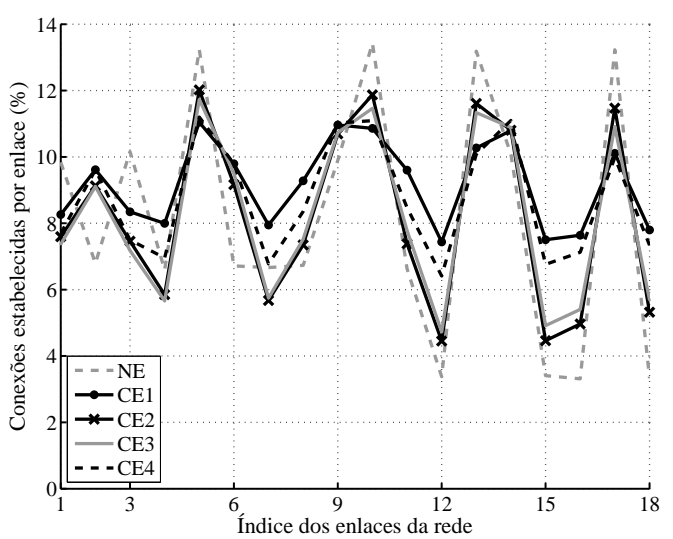

Fig. 9. Porcentagem de conexões estabelecidas para cada enlace para a topologia de 6 nós no $2^{\circ}$ cenário.

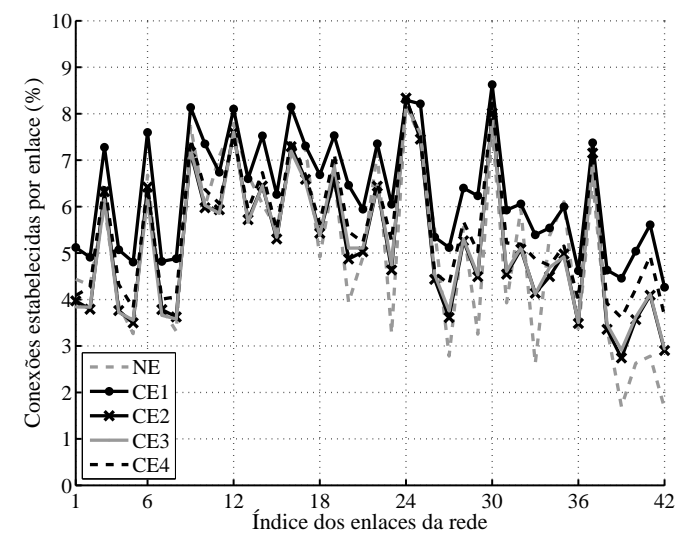

Fig. 10. Porcentagem de conexões estabelecidas para cada enlace para a topologia da rede NSF no $2^{\circ}$ cenário.

Os resultados mostram que a inserção da capacidade de agregação de tráfego levou à uma considerável melhoria na distribuição de carga da rede para ambas as topologias estudadas, considerando algumas funções. Para a topologia de seis nós, as funções CE2 e CE3 tiveram um desempenho pior, tendo em vista que a diminuição dos vales e picos foi percentualmente menor. A função CE4 manteve praticamente o mesmo desempenho e a função CE1 ocasionou uma distribuição mais uniforme dentre os dois cenários comparados. Já para a NSF, ocasionou uma melhora na distribuição de carga na rede para todas as funções custo analisadas, se comparados os resultados desse cenário com os resultados do primeiro cenário, com destaque para o desempenho mais significativo conseguido pela função CE1.

\section{CONCLUSÕES}

Dentre os benefícios da integração da agregação de tráfego com roteamento adaptativo comum se destaca a esperada diminuição no número de comprimentos de onda para se manter um dado número de bloqueios na rede. Tal característica se traduz em sistemas com menor custo se comparados aos que não utilizam a integração proposta. Por outro lado, se garante que a extensibilidade da rede projetada praticamente não será afetada, pois há a capacidade de se suportar um volume maior de tráfego.

Além disso, pode-se afirmar que os resultados dos cenários avaliados apresentam uma característica interessante: a de que a seleção da abordagem de roteamento a ser utilizada deve levar em consideração não apenas a probabilidade de bloqueio como métrica de escolha para o algoritmo a ser utilizado. Deve-se fazer uma ponderação entre o desempenho apresentado com relação à probabilidade de bloqueio e à distribuição das conexões pelos enlaces, objetivando, assim, um melhor custo benefício para o uso dos recursos da rede. Nessa busca por melhor uso dos recursos, a agregação de tráfego é uma ferramenta que, quando aliada ao roteamento adaptativo, gera tanto diminuição de probabilidade de bloqueio quanto uma distribuição mais uniforme dos recursos disponíveis na rede.

\section{REFERÊNCIAS}

[1] Hui Zang, Jason P. Jue, and Biswanath Mukherjee, "A Review of Routing and Wavelength Assignment Approaches for WavelengthRouted Optical WDM Networks", Optical Networks Magazine, Janeiro 2000.

[2] Iguatemi Eduardo da Fonseca, Uma Abordagem para Aprovisionamento $e$ Diferenciação de QoS Óptico na Presença de FWM em Redes Ópticas Transparentes, $\mathrm{PhD}$ thesis, Universidade Estadual de Campinas, Campinas, SP, Abril 2005.

[3] Rajiv Ramaswami and Kumar N. Sivarajan, Optical Networks: A Practical Perspective, Morgan Kaufmann Publishers, Inc., San Francisco, California, U.S.A., $2^{\mathrm{a}}$ edition, 2002

[4] Reinaldo Golmia Dante, Algoritmos de Roteamento e Atribuição de Comprimentos de Onda para as Redes Ópticas Inteligentes e Transparentes, $\mathrm{PhD}$ thesis, Universidade Estadual de Campinas, Campinas, SP, Novembro 2005.

[5] M. Brunato, R. Battiti, and E. Salvadori, "Dynamic Load Balancing in WDM Networks", Optical Networks Magazine, Setembro 2003.

[6] T. Fabry-Asztalos, N. Bhide, and K. M. Sivalingam, "Adaptive Weight Functions for Shortest Path Routing Algorithms for Multi-Wavelength Optical WDM Networks", in Proceedings of ICC 2000, New Orleans, LA, Junho 2000, vol. 3, pp. 1330-1334.

[7] Eytan Modiano and Philip J. Lin, "Traffic Grooming in WDM Networks", IEEE Communications Magazine, vol. 39, no. 7, pp. 124129, Julho 2001.

[8] Keyao Zhu and Biswanath Mukherjee, " On-line Approaches for Provisioning Connections of Different Bandwidth Granularities in WDM Mesh Networks", in Proc. Optical Fiber Communication Conference and Exhibit - OFC 2002, Março 2002, pp. 549- 551.

[9] Sashisekaran Thiagarajan and Arun K. Somani, "Capacity Fairness of WDM Networks with Grooming Capabilities", in Proceedings of SPIE, Setembro 2000, OptiComm 2000: Optical Networking and Communications, pp. 191-201.

[10] R. Srinivasan and Arun K. Somani, "A Generalized Framework for Analyzing Time-Space Switched Optical Networks", IEEE Journal on Selected Areas in Communications, vol. 20, pp. 202-215, Janeiro 2002.

[11] Sashisekaran Thiagarajan and Arun K. Somani, "Traffic Grooming for Survivable WDM Mesh Networks", in Proceedings of SPIE, Agosto 2001, vol. 4599 of OptiComm 2001: Optical Networking and Communications, pp. 54-65.

[12] L. A. Cox and J. Sanchez, "Cost Savings from Optimized Packing and Grooming of Optical Circuits: Mesh versus Ring Comparisons", SPIE Optical Networks Magazine, vol. 2, Maio 2001.

[13] Chunsheng Xin, Yinghua Ye, Sudhir Dixit, and Chunming Qiao, “ An Integrated Lightpath Provisioning Approach in Mesh Optical Networks", in Proc. Optical Fiber Communication Conference and Exhibit - OFC 2002, Março 2002, pp. 547- 549.

[14] Elio Salvadori, Traffic Engineering in Dynamic Optical Networks, PhD thesis, University of Trento, Trento - Itália, Fevereiro 2005. 